\title{
Outcomes among 6721 Hospitalized COVID-19 Patients across the New York City Public Hospital System: a Retrospective Cohort Study
}

J Gen Intern Med 36(4):1161-3

DOI: $10.1007 / \mathrm{s} 11606-020-06437-2$

(c) Society of General Internal Medicine 2021

\section{BACKGROUND}

Coronavirus-19 (COVID-19) has become a leading cause of death in the USA. New York City was heavily impacted by the virus, with hospitalizations and deaths disproportionately concentrated among lower income and minority communities. ${ }^{1}$ Clinical outcomes among such populations and within safety net care settings remain largely undescribed or methodologically constrained by short follow-up periods and large proportions of patients still hospitalized. ${ }^{2-5}$ Accordingly, we describe 30-day hospital outcomes among COVID-19 patients admitted to an 11-hospital public health care system in New York City.

\section{METHODS}

We conducted a retrospective cohort study of adults 18 years and older with laboratory-confirmed COVID-19 by real-time reverse transcriptase-polymerase chain reaction (RT-PCR) assay of nasopharyngeal swab specimens. The cohort included all admitted patients within 11 public hospitals in four boroughs of New York City during the period of the pandemic between March 1 and April 13, 2020. Data was collected through May 12 to allow this study period to enable a 30day follow-up for those patients admitted on April 13. This allowed avoidance of underreporting of mortality by including patients too early in their clinical course for meaningful outcome evaluation. We included only the most recent admission per patient, attributing transfers between study hospitals to the final hospital's borough.

Primary outcomes were hospital discharge, death or discharge to hospice, or ongoing hospitalization at 30 days after admission. By outcome, we described distributions of demographic and geographic characteristics, rates of intubation, and rates of renal replacement therapy (RRT); we further stratified patients by intubation status, RRT, and age. To characterize

Received September 17, 2020

Accepted December 9, 2020

Published online January 26, 2021 the degree to which mortality reflected patient goals to avoid life-sustaining treatments, we reviewed documented admission and final code status among patients who died or went to hospice to quantify the percent admitted as Do Not Resuscitate (DNR), transitioned to DNR during hospitalization, and maintained as full code up until study endpoint. Data were obtained from the electronic medical record. The study was exempted by the Biomedical Research Alliance of New York (BRANY) institutional review board.

\section{RESULTS}

During the study period, 6721 patients with laboratoryconfirmed COVID-19 were admitted at study hospitals, including 5113 (76.1\%) over age 50, 4130 (61.4\%) male, 2579 (38.4\%) non-English preferring, and 2786 (41.5\%) on Medicaid or self-pay (uninsured) (Table 1). During their hospitalization, $1683(25.0 \%)$ required intubation and 703 (10.5\%) RRT. By 30 days, 3984 (59.3\%) had been discharged, 2306 (34.3\%) had died or gone to hospice, and only 431 (6.4\%) remained hospitalized.

Outcomes varied by patient characteristics. Mortality strongly paralleled age (Table 1). Non-English and nonSpanish-preferring patients had higher mortality (43.7\%) than English- $(35.2 \%)$ or Spanish-preferring (30.2\%) ones, as did uninsured patients (34.9\%) compared to those with Medicaid $(22.0 \%)$ or commercial $(21.9 \%)$ insurance. Markedly higher mortality was observed among intubated patients $(72.0 \%)$ and those requiring RRT (57.2\%) (Table 1), compared to neither (21.1\%) (Table 2); for example, mortality among patients younger than 40 was $2.6 \%$ without intubation or RRT, climbing to $71.9 \%$ requiring both. One-quarter $(25.2 \%)$ of intubated patients requiring RRT remained in-hospital at 30 days. Among 2306 patients who died, 409 (17.7\%) were DNR upon admission, with the rest full code (39.7\%) or made DNR during hospitalization (42.6\%).

\section{DISCUSSION}

At 30 days, one in three patients admitted with COVID-19 at New York City public hospitals had expired or gone to hospice. Our use of 30-day outcomes minimizes mortality underreporting in other studies due to shorter follow-up 
Table 1 Patient Characteristics and 30-Day In-Hospital Outcomes Among Laboratory-Confirmed COVID-19 Patients Admitted to 11 Public Hospitals in New York City

\begin{tabular}{|c|c|c|c|c|}
\hline & \multirow{2}{*}{$\begin{array}{l}\text { Total, } N \\
(\%)^{*}\end{array}$} & \multicolumn{3}{|c|}{ Hospital outcome, $N(\%)^{\dagger}$} \\
\hline & & Discharged & $\begin{array}{l}\text { Died or } \\
\text { hospice }\end{array}$ & $\begin{array}{l}\text { In } \\
\text { hospital }\end{array}$ \\
\hline Total & $\begin{array}{l}6721 \\
(100.0)\end{array}$ & $3984(59.3)$ & $\begin{array}{l}2306 \\
(34.3)\end{array}$ & $431(6.4)$ \\
\hline \multicolumn{5}{|l|}{ Age, $N(\%)^{\dagger}$} \\
\hline $18-39$ & $\begin{array}{l}756 \\
(11.2)\end{array}$ & $633(83.7)$ & 89 (11.8) & $34(4.5)$ \\
\hline $40-49$ & $\begin{array}{l}852 \\
(12.7)\end{array}$ & $632(74.2)$ & $\begin{array}{l}156 \\
(18.3)\end{array}$ & $64(7.5)$ \\
\hline $50-59$ & $\begin{array}{l}1362 \\
(20.3)\end{array}$ & $917(67.3)$ & $\begin{array}{l}326 \\
(23.9)\end{array}$ & $119(8.7)$ \\
\hline $60-69$ & $\begin{array}{l}1526 \\
(22.7)\end{array}$ & $873(57.2)$ & $\begin{array}{l}519 \\
(34.0)\end{array}$ & $134(8.8)$ \\
\hline $70-79$ & $\begin{array}{l}1264 \\
(18.8)\end{array}$ & $598(47.3)$ & $\begin{array}{l}604 \\
(47.8)\end{array}$ & $62(4.9)$ \\
\hline $80+$ & $\begin{array}{l}961 \\
(14.3)\end{array}$ & $331(34.4)$ & $\begin{array}{l}612 \\
(63.7)\end{array}$ & $18(1.9)$ \\
\hline \multicolumn{5}{|l|}{ Sex, $N(\%)$} \\
\hline Female & $\begin{array}{l}2590 \\
(38.5)\end{array}$ & $1596(61.6)$ & $\begin{array}{l}852 \\
(32.9)\end{array}$ & $142(5.5)$ \\
\hline Male & $\begin{array}{l}4130 \\
(61.4)\end{array}$ & $2388(57.8)$ & $\begin{array}{l}1454 \\
(35.2)\end{array}$ & $288(7.0)$ \\
\hline $\begin{array}{l}\text { Unknown or } \\
\text { other }\end{array}$ & $1(0.0)$ & $0(0.0)$ & $0(0.0)$ & $1(100.0)$ \\
\hline \multicolumn{5}{|c|}{ Race/ethnicity, $N(\%)$} \\
\hline $\begin{array}{l}\text { Asian or } \\
\text { Pacific Islander }\end{array}$ & $385(5.7)$ & $213(55.3)$ & $\begin{array}{l}142 \\
(36.9)\end{array}$ & $30(7.8)$ \\
\hline Hispanic & $\begin{array}{l}751 \\
(11.2)\end{array}$ & $467(62.2)$ & $\begin{array}{l}232 \\
(30.9)\end{array}$ & $52(6.9)$ \\
\hline $\begin{array}{l}\text { Non-Hispanic } \\
\text { White }\end{array}$ & $609(9.1)$ & $306(50.2)$ & $\begin{array}{l}283 \\
(46.5)\end{array}$ & $20(3.3)$ \\
\hline Non-Hispanic & 2084 & $1286(61.7)$ & 686 & $112(5.4)$ \\
\hline $\begin{array}{l}\text { Black } \\
\text { Other or }\end{array}$ & $(31.0)$ & & $(32.9)$ & \\
\hline $\begin{array}{l}\text { Other or } \\
\text { unknown }\end{array}$ & $\begin{array}{l}2892 \\
(43.0)\end{array}$ & $1712(59.2)$ & $\begin{array}{l}963 \\
(33.3)\end{array}$ & $217(7.5)$ \\
\hline \multicolumn{5}{|c|}{ Preferred language, $N(\%)$} \\
\hline English & $\begin{array}{l}4142 \\
(61.6)\end{array}$ & $2452(59.2)$ & $\begin{array}{l}1460 \\
(35.2)\end{array}$ & $230(5.6)$ \\
\hline Spanish & $\begin{array}{l}2082 \\
(31.0)\end{array}$ & $1287(61.8)$ & $\begin{array}{l}629 \\
(30.2)\end{array}$ & $166(8.0)$ \\
\hline $\begin{array}{l}\text { Other or } \\
\text { unknown }\end{array}$ & $497(7.4)$ & $245(49.3)$ & $\begin{array}{l}217 \\
(43.7)\end{array}$ & $35(7.0)$ \\
\hline \multicolumn{5}{|l|}{ Borough, $N(\%)$} \\
\hline & $(27.2)$ & & $(32.8)$ & \\
\hline Brooklyn & $\begin{array}{l}1779 \\
(26.5)\end{array}$ & $993(55.8)$ & $\begin{array}{l}695 \\
(39.1)\end{array}$ & $91(5.1)$ \\
\hline Manhattan & $\begin{array}{l}1495 \\
(22.2)\end{array}$ & $945(63.2)$ & $\begin{array}{l}403 \\
(27.0)\end{array}$ & $147(9.8)$ \\
\hline Queens & $\begin{array}{l}1621 \\
(24.1)\end{array}$ & $926(57.1)$ & $\begin{array}{l}609 \\
(37.6)\end{array}$ & $86(5.3)$ \\
\hline \multicolumn{5}{|c|}{ Primary payer, $N(\%)$} \\
\hline Medicare & $\begin{array}{l}2641 \\
(39.3)\end{array}$ & $1165(44.1)$ & $\begin{array}{l}1345 \\
(50.9)\end{array}$ & $131(5.0)$ \\
\hline Medicaid & $\begin{array}{l}2291 \\
(34.1)\end{array}$ & $1598(69.8)$ & $\begin{array}{l}505 \\
(22.0)\end{array}$ & $188(8.2)$ \\
\hline Commercial & $\begin{array}{l}1270 \\
(18.9)\end{array}$ & 911 (71.7) & $\begin{array}{l}278 \\
(21.9)\end{array}$ & $81(6.4)$ \\
\hline $\begin{array}{l}\text { Self-pay } \\
\text { (uninsured) }\end{array}$ & $495(7.4)$ & $293(59.2)$ & $\begin{array}{l}173 \\
(34.9)\end{array}$ & $29(5.9)$ \\
\hline Other & $24(0.4)$ & $17(70.8)$ & $5(20.8)$ & $2(8.3)$ \\
\hline \multicolumn{5}{|l|}{ Intubated, $N(\%)$} \\
\hline Yes & $\begin{array}{l}1683 \\
(25.0)\end{array}$ & $177(10.5)$ & $\begin{array}{l}1212 \\
(72.0)\end{array}$ & $\begin{array}{l}294 \\
(17.5)\end{array}$ \\
\hline No & $\begin{array}{l}5038 \\
(75.0)\end{array}$ & $3807(75.6)$ & $\begin{array}{l}1094 \\
(21.7)\end{array}$ & $137(2.7)$ \\
\hline \multicolumn{5}{|c|}{ Renal replacement, $N(\%)$} \\
\hline Yes & $\begin{array}{l}703 \\
(10.5)\end{array}$ & $174(24.8)$ & $\begin{array}{l}402 \\
(57.2)\end{array}$ & $\begin{array}{l}127 \\
(18.1)\end{array}$ \\
\hline No & $\begin{array}{l}6018 \\
(89.5)\end{array}$ & $3810(63.3)$ & $\begin{array}{l}1904 \\
(31.6)\end{array}$ & $304(5.1)$ \\
\hline
\end{tabular}

*Percentages reported in aggregate are of column totals, ${ }^{\dagger}$ Percentages reported by endpoint are of row totals. ${ }^{7}$ Discharge includes patients discharged to home, skilled nursing, rehabilitation, long-term acute care, or other intermediate care levels
Table 2 Thirty-Day In-Hospital Outcomes Among Recipients and Non-recipients of Intubation and Renal Replacement Therapy Among Patients with COVID-19 Admitted to 11 Public Hospitals in New York City

\begin{tabular}{|c|c|c|c|}
\hline & Hospital outcome, & $(\%)^{*}$ & \\
\hline & $\begin{array}{l}\text { Discharged }{ }^{\dagger}, N= \\
3984\end{array}$ & $\begin{array}{l}\text { Died or hospice, } \\
N=2306\end{array}$ & $\begin{array}{l}\text { In hospital, } \\
N=\mathbf{4 3 1}\end{array}$ \\
\hline Intubated & & & \\
\hline Renal re & placement therapy & & \\
\hline Total & $18(4.0)$ & $320(70.8)$ & $114(25.2)$ \\
\hline Age, $N$ & $(\%)$ & & \\
\hline $18-$ & $3(9.4)$ & $23(71.9)$ & $6(18.8)$ \\
\hline $40-$ & $3(6.0)$ & $30(60.0)$ & $17(34.0)$ \\
\hline $50-$ & $4(3.6)$ & $73(65.8)$ & $34(30.6)$ \\
\hline 60 & $5(3.2)$ & $112(71.3)$ & $40(25.5)$ \\
\hline $70-$ & $3(3.8)$ & $64(80.0)$ & $13(16.3)$ \\
\hline $80+$ & $0(0.0)$ & $18(81.8)$ & $4(18.2)$ \\
\hline No rena & replacement therapy & & \\
\hline Total & $159(12.9)$ & $892(72.5)$ & $180(14.6)$ \\
\hline Age, $N$ & $(\%)$ & & \\
\hline $18-$ & $36(37.5)$ & $49(51.0)$ & $11(11.5)$ \\
\hline $40-$ & 30 (21.6) & $81(58.3)$ & $28(20.1)$ \\
\hline $50-$ & $37(15.2)$ & $155(63.8)$ & $51(21.0)$ \\
\hline $60-$ & $34(10.3)$ & $239(72.2)$ & $58(17.5)$ \\
\hline 70 & $17(6.1)$ & $238(85.0)$ & $25(8.9)$ \\
\hline $80+$ & $5(3.5)$ & $130(91.5)$ & $7(4.9)$ \\
\hline Not intub & ited & & \\
\hline Renal re & placement therapy & & \\
\hline $\begin{array}{l}\text { Total } \\
\text { Age, } \Lambda\end{array}$ & $156(62.2)$ & $82(32.7)$ & $13(5.2)$ \\
\hline $\begin{array}{c}\text { Age, } \Lambda \\
18-\end{array}$ & $\begin{array}{l}(\%) \\
11(91.7)\end{array}$ & $1(8.3)$ & $0(0.0)$ \\
\hline $3940-$ & $26(867)$ & $2(67)$ & $2(67)$ \\
\hline 49 & & & \\
\hline${ }_{59}^{50-}$ & $42(71.2)$ & $13(22.0)$ & $4(6.8)$ \\
\hline $60-$ & $38(62.3)$ & $20(32.8)$ & $3(4.9)$ \\
\hline 70 & $33(54.1)$ & $25(41.0)$ & $3(4.9)$ \\
\hline $80+$ & $6(21.4)$ & $21(75.0)$ & $1(3.6)$ \\
\hline No rena & replacement therapy & & \\
\hline Total & $3651(76.3)$ & $1012(21.1)$ & $124(2.6)$ \\
\hline Age, $\Lambda$ & $(\%)$ & & \\
\hline $18-$ & $583(94.6)$ & $16(2.6)$ & $17(2.8)$ \\
\hline $40-$ & $573(90.5)$ & $43(6.8)$ & $17(2.7)$ \\
\hline $50-$ & $834(87.9)$ & $85(9.0)$ & $30(3.2)$ \\
\hline $60-$ & $796(81.5)$ & $148(15.1)$ & $33(3.4)$ \\
\hline $70-$ & $545(64.7)$ & $277(32.9)$ & $21(2.5)$ \\
\hline $80+$ & $320(41.6)$ & $443(57.6)$ & $6(0.8)$ \\
\hline
\end{tabular}

*Percentages reported by endpoint are of row totals. ${ }^{\dagger}$ Discharge includes patients discharged to skilled nursing, rehabilitation, long-term acute care, or other intermediate care levels

periods or high proportions of patients remaining in-hospital. $^{2,3}$ Higher rates of intubation and RRT in the hospitals studied than elsewhere may suggest more advanced disease 
or greater underlying medical risk among our patient population. $^{2-5}$ Furthermore, greater uninsurance and underinsurance, linguistic diversity, and social risk among public hospital patients may have provoked delays seeking care, worsening outcomes. Finally, a large proportion of patients who died — nearly one in five - were admitted as DNR, warranting comparison across care settings. There were several limitations to this descriptive study: the lack of data on known cause of death; therapeutics given did not allow for statistical analysis of the cohort to determine impacts of such outcomes. Despite this, COVID-19's substantial impact on public hospitals suggests the need to identify solutions to increase access to timely care and mitigate outcome disparities among lower income and minority populations. ${ }^{6}$

Acknowledgments: The authors wish to acknowledge the following members of the NYC Health + Hospitals COVID-19 Emergency Medicine Investigator Group including Raj Gulati, MD of NYC Health + Hospitals/Bellevue, Jeanette Haslett, MD of NYC Health + Hospitals/Harlem, Getawworku Hassen, MD, PhD of NYC Health + Hospitals/Metropolitan, Jordan Jeong, DO of NYC Health + Hospitals/Coney Island, and Richard Sinert, DO of NYC Health + Hospitals/Kings County for their contributions to the writing and revision of this research report.

Nicholas Caputo, $\mathrm{MD}, \mathrm{MSc}^{1}$

John Zhang, $\mathrm{Ph} \mathrm{D}^{2}$

Robert Chin, $M D^{3}$

Chad Meyers, $M D^{4}$

Shaw Natsui, MD, MPA ${ }^{5}$

David M. Silvestri, MD, MBA, $M_{H}{ }^{5}$

Jason Z. D'Amore, MD, MBA ${ }^{6}$

${ }^{1}$ Department of Emergency Medicine, NYC Health + Hospitals/Lincoln,

Bronx, NY, USA

${ }^{2}$ NYC Health + Hospitals/Lincoln,

Bronx, NY, USA
${ }^{3} \mathrm{NYC}$ Health + Hospitals/Woodhull,

Brooklyn, NY, USA

${ }^{4} \mathrm{NYC}$ Health + Hospitals/Elmhurst, Queens, NY, USA

${ }^{5} \mathrm{NYC}$ Health + Hospitals/Central Office,

New York, NY, USA

${ }^{6} \mathrm{NYC}$ Health + Hospitals/Jacobi,

Bronx, NY, USA

Corresponding Author: Nicholas Caputo, MD, MSc; Department of Emergency Medicine, NYC Health + Hospitals/Lincoln, Bronx, NY, USA (e-mail: nicholas.caputo@nychhc.org).

\section{Compliance with Ethical Standards:}

Conflict of Interest: The authors declare that they do not have a conflict of interest.

\section{REFERENCES}

1. Wadhera RK, Wadhera $\mathbf{P}$, Gaba $\mathbf{P}$, et al. Variation in COVID-19 hospitalizations and deaths across New York City boroughs. JAMA. 2020; 323(21): 2912-5.

2. Goyal P, Choi JJ, Pinheiro LC, et al. Clinical characteristics of Covid-19 in New York City. $N$ Engl $J$ Med. 2020: NEJMc2010419.

3. Richardson S, Hirsch JS, Narasimhan M, et al. Presenting characteristics, comorbidities, and outcomes among 5700 patients hospitalized with COVID-19 in the New York City area. JAMA. 2020; 323(20): 2052-9.

4. Petrilli, CM, Jones SA, Yang J, et al. Factors associated with hospital admission and critical illness among 5279 people with coronavirus disease in 2019 in New York City: prospective cohort study. BMJ. 2020; 369: m1966.

5. Argenziano, MG, Bruce SL, Slater CL, et al. Characterization and clinical course of 1000 patients with coronavirus disease 2019 in New York: retrospective case series. BMJ. 2020; 369: m1996.

6. Bibbins-Domingo $\mathbf{K}$. This time must be different: disparities during the COVID-19 pandemic. Ann Intern Med. 2020; M20-2247.

Publisher's Note: Springer Nature remains neutral with regard to jurisdictional claims in published maps and institutional affiliations. 